\title{
Proper Motion of Water Masers Associated with IRAS 21391+5802: Bipolar Outflow and an AU\# Scale Dusty Circumstellar Shell
}

\section{Citation}

Patel, Nimesh A., Lincoln J. Greenhill, James Herrnstein, Qizhou Zhang, James M. Moran, Paul T. P. Ho, and Paul F. Goldsmith. 2000. "Proper Motion of Water Masers Associated with IRAS 21391+5802: Bipolar Outflow and an AU\#Scale Dusty Circumstellar Shell." The Astrophysical Journal 538 (1) (July 20): 268-274. doi:10.1086/309126.

\section{Published Version}

$10.1086 / 309126$

\section{Permanent link}

http://nrs.harvard.edu/urn-3:HUL.InstRepos:32094220

\section{Terms of Use}

This article was downloaded from Harvard University's DASH repository, and is made available under the terms and conditions applicable to Other Posted Material, as set forth at http:// nrs.harvard.edu/urn-3:HUL.InstRepos:dash.current.terms-of-use\#LAA

\section{Share Your Story}

The Harvard community has made this article openly available.

Please share how this access benefits you. Submit a story.

Accessibility 


\title{
Proper motion of water masers associated with IRAS 21391+5802: Bipolar Outflow and an AU-scale Dusty Circumstellar Shell
}

\author{
Nimesh A. Patel ${ }^{1}$ \\ (npatel@cfa.harvard.edu) \\ Lincoln J. Greenhill ${ }^{1}$ \\ (lgreenhill@cfa.harvard.edu) \\ James Herrnstein ${ }^{2}$ \\ (jherrnstein@nrao.edu) \\ Qizhou Zhang ${ }^{1}$ \\ (qzhang@cfa.harvard.edu) \\ James M. Moran ${ }^{1}$ \\ (jmmoran@cfa.harvard.edu) \\ Paul T. P. Ho ${ }^{1}$ \\ (pho@cfa.harvard.edu) \\ Paul F. Goldsmith ${ }^{3}$ \\ (pfg@astrosun.tn.cornell.edu)
}

\begin{abstract}
We present VLBA observations of water maser emission associated with the star forming region IRAS $21391+5802$, which is embedded in a bright rimmed cometary globule in IC1396. The angular resolution of the maps is $\sim 0.8$ mas, corresponding to a spatial resolution of $\sim 0.6 \mathrm{AU}$, at an estimated distance of $750 \mathrm{pc}$. Proper motions are derived for 10 maser features identified consistently over three epochs, which were separated by intervals of about one month. The
\end{abstract}

\footnotetext{
${ }^{1}$ Harvard-Smithsonian Center for Astrophysics, 60 Garden St., Cambridge, MA 02138

${ }^{2}$ National Radio Astronomy Observatory, P.O. Box 0, Socorro, NM 87801

${ }^{3}$ National Astronomy and Ionosphere Center, Cornell University, Department of Astronomy, Ithaca, NY 14853
} 
masers appear in four groups, which are aligned linearly on the sky, roughly along a northeast-southwest direction, with a total separation of $\sim 520$ AU $\left(\sim 00^{\prime \prime} 7\right)$. The $3 \mathrm{D}$ velocities of the masers have a maximum value of $\sim 42 \mathrm{~km} \mathrm{~s}^{-1}$ $\left(\sim 9 \mathrm{AU} \mathrm{yr}^{-1}\right)$. The average error on the derived proper motions is $\sim 4 \mathrm{~km} \mathrm{~s}^{-1}$. The overall pattern of proper motions is indicative of a bipolar outflow. Proper motions of the masers in a central cluster, with a projected extent of $\sim 20 \mathrm{AU}$, show systematic deviations from a radial outflow. However, we find no evidence of Keplerian rotation, as has been claimed elsewhere. A nearly circular loop of masers lies near the middle of the cluster. The radius of this loop is $1 \mathrm{AU}$ and the line-of-sight velocities of the masers in the loop are within $2 \mathrm{~km} \mathrm{~s}^{-1}$ of the systemic velocity of the region. These masers presumably exist at the radial distance where significant dust condensation occurs in the outflow emanating from the star.

Subject headings: ISM: jets and outflows — masers — stars: formation

\section{Introduction}

An important phase in the evolution of young stellar objects (YSO) is the one during which they exhibit energetic mass outflows, and simultaneous accretion of material onto the star-forming core. Since the discovery of bipolar outflows (Snell et al. 1980), much progress has been made in the study of the large scale and phenomenological characteristics of such outflows (e.g. Bachiller 1996), but relatively fewer studies have probed the regions within $\sim 100$ AU of the YSOs, where the winds that drive the outflows, originate (e.g., Shu et al. 1994).

A significant fraction of all YSOs exhibit water maser emission, and the maser luminosity appears to be correlated with the mechanical luminosity of the outflows (Felli et al. 1992). The maser emission is thought to arise from dust-laden shocked gas close to the exciting YSOs, where densities are $\sim 10^{9} \mathrm{~cm}^{-3}$ and temperatures are $\sim 400 \mathrm{~K}$ (e.g. Elitzur et al. 1989). Since such conditions are likely to be highly localized, and since the velocity coherence required for building up large path lengths for the maser amplification is also likely to occur over relatively smaller regions, the water maser emission provides an excellent tool for probing the kinematics of the gas with high angular resolution ( $\sim 1$ mas). Radio interferometric observations of the water maser emission and measurements of maser proper motions have been carried out mostly towards high-mass star forming regions such as Orion-KL, W49N, W51 Main, W51 North, Sgr B2 and W3 (OH) (Genzel et al. 1981a; 
Gwinn et al. 1992; Genzel et al. 1981b; Schneps et al. 1981; Reid et al. 1988; Alcolea et al. 1993). Similar studies of low and intermediate-mass $\left(<10 \mathrm{M}_{\odot}\right)$ star forming regions with very high spatial resolution are only now becoming available (Claussen et al. 1998, Furuya et al. 1999, Wootten et al. 1999).

IRAS $21391+5802$ is an infrared source embedded in a bright-rimmed globule appearing on the northern periphery of the giant H II region IC1396. Several observational studies of this object at various wavelengths suggest that it is an intermediate-mass star forming region (Sugitani et al. 1989, Wilking et al. 1993, Patel et al. 1995, Cesaroni et al. 1999). Its far-infrared luminosity is $235 \mathrm{~L}_{\odot}$ (Saraceno et al. 1996). Water maser emission from this source was first detected by Felli et al. (1992) and interferometric observations were made by Tofani et al. (1995) using the NRAO' Very Large Array (VLA) at a resolution of 0".1 resolution. These observations revealed a linear distribution of masers, with a systematic velocity gradient along its length. More recently, Slysh et al. (1999) reported single-epoch observations of these masers with mas angular resolution. However, interpretation of the kinematics has remained ambiguous without proper motion data.

We present here the results of a multi-epoch program of interferometric observations of the water maser emission from IRAS21391+5802. In the next section, we summarize the observational details, followed by results and our interpretation using a simple model of a bipolar outflow.

\section{Observations and data reduction}

We observed the $6_{16}-5_{23}$ maser line at $22.23508 \mathrm{GHz}$, using the Haystack $37 \mathrm{~m}$ telescope, the NRAO 140-foot antenna at Greenbank, WV, the VLA in its largest and smallest configurations, and the NRAO Very Large Baseline Array (VLBA). The single-dish observations in 1994-1995 provided a time-series of spectra, while we determined absolute astrometric positions of the masers using the VLA observations.

The VLBA observations were made for 6 hours on 1996 March 17, 1996 April 20 and 1996 May 19. We observed a single $4 \mathrm{MHz}$ bandpass in left circular polarization, covering -27 to $+27 \mathrm{~km} \mathrm{~s}^{-1}$ in velocity, and divided into 256 channels of $0.21 \mathrm{~km} \mathrm{~s}^{-1}$ each. The systemic velocity of the source, based on observations of ${ }^{13} \mathrm{CO} \mathrm{J}=1-0$ emission, with a velocity resolution of $0.7 \mathrm{~km} \mathrm{~s}^{-1}$, is about $0 \mathrm{~km} \mathrm{~s}^{-1}$ with respect to the Local Standard of

\footnotetext{
${ }^{1}$ The National Radio Astronomy Observatory is a facility of the National Science Foundation operated under a cooperative agreement with Associated Universities, Inc.
} 
Rest (LSR) (Patel et al. 1995). The data were correlated at the NRAO Array Operation Center at Socorro, NM with an averaging time of 1.3 seconds, which provided a field-of-view of $\sim 1^{\prime \prime}$ for the whole array.

The data were reduced and imaged with standard techniques using the NRAO AIPS package. Observations of 3C345, 1739+522, 2007+777, BL LAC, and 3C454.3 provided delay and phase calibration. The sources 3C345 and BL Lac were used for bandpass calibration. The synthesized beam in all three epochs was $\sim 0.8 \times 0.4$ mas at a position angle of $\sim 20^{\circ}$. The rms noise in the final images was $\sim 6$ mJy in all 3 epochs.

Initially, maps with reduced $1 \times 1$ mas resolution were made to identify the approximate locations of all the maser spots in a $1^{\prime \prime} \times 1^{\prime \prime}$ field. We define a maser "spot" as emission occurring in a given velocity channel. A maser "feature" is a collection of spots over contiguous channels. Maps with the highest resolution were made for selected regions and two dimensional elliptical Gaussians were fitted to each identified maser spot. The positions of the masers were measured with respect to the spot at $-14.6 \mathrm{~km} \mathrm{~s}^{-1}$ in maser feature A, which was used as a reference for self-calibration in all three epochs (i.e., for calibration of atmospheric path length fluctuations). The resulting position measurements have a mean formal uncertainty of $\sim 10 \mu$ as. For each spot, the adopted uncertainty is the larger of 1 ) the theoretical uncertainty from the beam-width and the signal-to-noise ratio, and 2) the measured uncertainty from the profile fit. Contributions to the position uncertainties from the interferometric calibration (e.g., clock, astrometry, or baseline errors) were negligible. For each epoch, maser spot positions and line-of-sight velocities were inspected graphically to identify distinct features. The number of identifiable maser features in the three epochs was 21,19 , and 20 , respectively.

Ten maser features persisted in all three epochs, with mean line-of-sight velocities changing by less than $0.5 \mathrm{~km} \mathrm{~s}^{-1}$ ( $\frac{1}{2}$ linewidth). Proper motions were estimated by a least-squares fit to position as a function of time, with weights that were inversely proportional to the square of the uncertainties in position measurement. The weights were scaled to achieve unit reduced $\chi^{2}$. A noise floor of $80 \mu$ as was added in quadrature to the uncertainties to account for possible unresolved structure within the maser spots. The noise floor corresponded approximately to the observed wander in the motion of the maser features about a straight-line trajectory. Proper motions for features observed at only two epochs were discarded because their deviations from the straight-line motion could not be estimated. 


\section{Results}

A time-series of spectra obtained during the time-monitoring observations of the water maser emission from IRAS 21391+5802 are shown in Fig. 1. The epochs are separated by $\gtrsim 1$ month. The spectral-lines change significantly from epoch to epoch. The center velocities of some lines seem to change as well. However, data obtained over shorter time intervals ( $\leq 2$ weeks; not shown here), do not show any significant changes.

The velocity structure of the spectra are symmetric around the systemic velocity of $\sim 0 \mathrm{~km} \mathrm{~s}^{-1}$, but the VLA images show that the $10 \mathrm{~km} \mathrm{~s}^{-1}$ emission is offset southeast by $\sim 10^{\prime \prime}$ (7500 AU) (see also Tofani et al. 1995). Earlier interferometric observations of thermal continuum emission at $3 \mathrm{~mm}$, and $\mathrm{C}^{18} \mathrm{O} \mathrm{J}=1-0$ line emission made by Wilking et al. (1993), show an extension from the central peak towards this $10 \mathrm{~km} \mathrm{~s}^{-1}$ maser feature. We suggest that this is a separate star forming condensation, unrelated to the rest of the masers in our map. Wilking et al. (1993) identified four near-infrared sources towards IRAS21391+5802. IRS2 (Wilking et al. 1993) which is associated with the $3 \mathrm{~mm}$ peak radio continuum emission, is closest to the water masers shown in Fig. 2. In the observations by Wilking et al. (1993), there does not appear to be an infrared source associated with the $10 \mathrm{~km} \mathrm{~s}^{-1}$ maser feature that is offset 10" from the bulk of the maser emission.

Fig. 2 shows the results of our VLBA observations over the three epochs. The groups of maser features labeled A, C and D are isolated. These masers have line-of-sight velocities differing by more than $\pm 4 \mathrm{~km} \mathrm{~s}^{-1}$ from the systemic velocity. A and D are the most extremely blue and red-shifted masers, respectively, and they also appear at the extrema of the distribution of masers on the sky. Most of the masers in B appear to be aligned along an eastwest line near $\Delta \delta=0$.' 17 . We refer to these hereafter as the "inner" masers. The integrated intensity maps of the inner masers are shown in Fig. 3. From an analysis of fringe rates, we estimate that the position of the reference emission is $\alpha_{J 2000}=21^{h} 40^{m} 41^{s} .791 \pm 0^{s} .004, \delta_{J 2000}=58^{\circ} 16^{\prime} 11^{\prime \prime} .737 \pm 0^{\prime \prime} 030$. Our VLA A array observations made on 6 July 1995, add information on the variability of maser emission in this region. The maser features $\mathrm{A}$ and $\mathrm{B}$ appear roughly in the same positions and line-of-sight velocities as in the VLBA observations. Two different maser features appear in the VLA A array observations. One at a position offset of $(0.41,0$ ". 11$)$ with a line-of-sight

velocity of $-8 \mathrm{~km} \mathrm{~s}^{-1}$ and the other at $(0$. " 63,0 "' 25$)$ and velocity of $22 \mathrm{~km} \mathrm{~s}^{-1}$. Maser features $\mathrm{C}$ and D do not appear in the VLA map. Because the position uncertainties are about 0.'1 in the VLA map, registered with respect to the VLBA map, the VLA data are not useful in estimation of motions.

The proper motions in clumps A, C and D are suggestive of largely radial outflow from a center of expansion in clump B. Within clump B there is a roughly circular loop of masers 
at $\Delta \alpha=0.515-0 . .519$ and $\Delta \delta=0.16-0$.'17. The line-of-sight velocities of these masers are the closest to the systematic velocity of the region, and the velocities in the plane of the sky are consistent with a radial outflow. From these results we propose that the loop signifies the radius of dust sublimation in a circumstellar shell of outward flowing stellar wind material. Maser emission is beamed along the line-of-sight by the long gain paths tangent to the edge of the relatively dust-free inner cavity.

From each feature, we have subtracted the mean measured proper motion for the whole source, to reduce the effect of the proper motion of the reference feature. In principle, an arbitrary velocity vector may be added to the proper motions since they are obtained from positions to the feature at $-14.6 \mathrm{~km} \mathrm{~s}^{-1}$. If we add a proper motion of $6.8 \mathrm{~km} \mathrm{~s}^{-1}$ at a position angle of $29^{\circ}$ to each maser feature, then the two proper motion vectors associated with the loop become radial and outward. However, with the addition of this largely northward motion, the proper motions of the three easternmost features in B deviate significantly from a radial outflow. Although the assignment of zero net proper motion may be somewhat arbitrary, it is the most straight-forward way to compensate at least partly for the relatively large apparent motion of the reference maser feature.

\section{Discussion}

The positions and 3D space velocities in Fig. 2 clearly show that systematic motions dominate over turbulent motions in this system. The masers trace bulk gas kinematics within $0 . \prime 6$ or $500 \mathrm{AU}$ of the possible YSO. In principle, within such close proximity, both infall along an accreting circumstellar disk and an orthogonal bipolar outflow may be anticipated (e.g., Shu et al. 1987). Relying on interpretation of spectra and a single epoch VLBA observation of these masers, Slysh et al. (1999) conclude that the maser kinematics

might represent Keplerian rotation of a circumstellar disk around a YSO. However, in such a scenario the masers with line-of-sight velocity nearest the systemic velocity should show the largest proper motions. Our data clearly rule out a disk model and support a bipolar outflow model.

The loop of masers that we propose pinpoints the possible YSO (Figs. 2 \& 3) has a radius of $\sim 1 \mathrm{AU}$. The bolometric luminosity of IRAS $21391+5802$ is $235 \mathrm{~L}_{\odot}$, implying a mass of 3 to $5 \mathrm{M}_{\odot}$. Gravitationally bound gas within a radius of a few AU of a $3 \mathrm{M}_{\odot}$ object should exhibit orbital velocities of a few tens of $\mathrm{km} \mathrm{s}^{-1}$. However, all the features in the loop have line-of-sight velocities that are an order of magnitude less. Furthermore, two of the features in the loop for which proper motions could be determined show 3D space velocities of only $\sim 5 \mathrm{~km} \mathrm{~s}^{-1}$. If the masers lie at about the radius where substantial 
condensation of dust grain occurs, then this is also the radius at which acceleration of the outflow by radiation pressure begins; the low 3D space velocities are reasonable and may be upper limits on bulk outflow speeds closer to the YSO. Since the earliest observations of the maser source (Felli et al. 1992, Brand et al. 1994) the maser emission at line-of-sight velocities corresponding to the loop masers has been persistent, in contrast to the higher velocity maser features, which apparently lie downstream in the outflow. The masers in the loop may thus represent a standing pattern in outward flowing material, wherein masers are born, track outflowing and cooling material, and fade, to be replaced by new masers at smaller radii. Masers downstream are products of shocks and local heating possibly in collisions between the outflow and ambient media.

The spectral energy distribution of the infrared emission from IRAS 21391+5802 has been studied by Correia et al. (1999). According to their model, the temperature of the gas at a distance of $1 \mathrm{AU}$ from the YSO is predicted to be about $1800 \mathrm{~K}$. This is in excess by at least a factor of two higher than the temperature expected from the occurrence of the water masers in the loop. However, the calculated temperature depends sensitively on grain properties such as size. If the grains are larger than $1 \mu \mathrm{m}$ in size, the dust envelope would be optically thin very close to the star, and lower temperatures would be possible $(\sim 1000 \mathrm{~K})$.

The 3D space velocities of masers A, C and D shown in Fig. 2 indicate an outflow away from the inner masers (B). To estimate the position and inclination angles of the outflow, we fit a simple model to the data in which we assume an outflow velocity that is linearly proportional to the distance from the outflow center, with a proportionality constant, $k$. We choose a coordinate system with its origin at the center of the loop. The purpose of this model is only to estimate the position and inclination angles and the value of $k$. A straight line fitted to the observed maser positions alone provides the position angle $\phi \approx 70^{\circ}$. From the positions and $3 \mathrm{D}$ velocities, we obtain an inclination angle $\theta \approx 70^{\circ}$ with respect to the line of sight, $k$, and $z_{i}$, the distance of each maser spot along the line of sight, and $k \approx 5 \times 10^{-10} s^{-1}\left(0.07 \mathrm{~km} \mathrm{~s}^{-1} \mathrm{AU}^{-1}\right)$. The inclination and position angle are difficult to measure well because the outflow appears to have an opening angle of at least $40^{\circ}$, judging from the motions and line-of-sight velocities of $\mathrm{C} \& \mathrm{D}$, and from the distribution of maser spots in B.

The fitted value of $k$ implies an overall e-folding time of $\sim 65$ yrs. The dynamical times obtained from the de-projected radii of the masers and the 3D space velocities are 43, 38 and $14 \mathrm{yrs}$ for the masers A, D and C. The inner masers (B) have a dynamical time of only 2 to 9 yrs. These different dynamical times are inconsistent with the masers being ejected ballistically at the same time but with different initial velocities. Instead, there is possibly true acceleration in the flow as a function of distance away from the YSO. 
Another possibility is that the flow is ballistic but episodic. However, as noted earlier, maser emission at velocities close to the systemic velocity (corresponding to B \& L) seems to have persisted since the earliest observations in 1988 (Brand et al. 1994). Thus, the mass outflow represented by the clumps of gas that are masing today, may be a continuous process, rather than a consequence of a singular event of mass ejection.

\section{Conclusions}

We have detected proper motion of 10 water maser features associated with the star-forming region IRAS $21391+5802$. The water masers trace a bipolar outflow from an intermediate mass young stellar object. We estimate position and inclination angles of $\sim 70^{\circ}$ for the outflow axis, though the opening angle may exceed $40^{\circ}$. The maximum observed 3D space velocity of the masers is $42 \mathrm{~km} \mathrm{~s}^{-1}$. The observed 3D space velocities of the masers suggest the presence of acceleration within the outflow, but we cannot rule out episodic ballistic ejection. Near the center of the flow, there is a roughly circular loop of masers with a radius of $\sim 1$ AU. The masers in this loop are most likely to be tangentially amplified within a shell of dense gas surrounding the YSO. Because there is no evidence for rotation in the source (as in a disk), the shell probably comprises wind material alone. However, dynamics of the IRAS 21391+5802 region are poorly sampled, especially in the loop. Additional observations may still reveal some rotation, or even infall.

We thank Lee Hartmann, Nuria Calvet, T. K. Sridharan and Masao Saito for helpful discussions. We are grateful to Shoshana Rosenthal for help with the management of the

Alpha workstations and AIPS in the Radio and Geoastronomy division at the Center for Astrophysics.

\section{REFERENCES}

Alcolea, J., Menten, K. M., Moran, J. M. \& Reid, M. J., 1993, in Astrophysical Masers, Lecture Notes in Physics, Vol. 412, The Proper Motions of the $\mathrm{H}_{2} \mathrm{O}$ Masers Near W3 $(\mathrm{OH})$, ed. A. Clegg and G. Nedoluha, (Berlin: Springer-Verlag), 225

Bachiller, R. 1996, ARA\&A, 34,111

Brand, J., et al. 1994, A\&AS, 103, 541

Cesaroni, R., Felli, M. \& Walmsley, C. M. 1999, A\&AS, 136, 333

Claussen, M. J., Marvel, K. B., Wootten, A. \& Wilking, B. A. 1998, ApJ, 507, L79 
Correia, J. C, Griffin, M. \& Saraceno, P., 1999, in The Universe as seen by ISO, ed. P. Cox \& M. F. Kessler, (Noordwijk: ESA Publications Division), 479

Elitzur, M., Hollenbach, D. J. \& McKee, C. F. 1989, ApJ, 346, 983

Felli, M., Palagi, F., Tofani, G. 1992, A\&A, 255, 293

Furuya, R., et al. 1999, in Star Formation 1999, ed. T. Nakamoto, (Nagoya: Nagoya University), in press

Genzel, R., Reid, M. J., Moran, J. M. \& Downes, D. 1981a, ApJ, 244, 884

Genzel, R., et al. 1981b, ApJ, 247, 1039

Gwinn, C. R., Moran, J. M \& Reid, M. J. 1992, ApJ, 393, 149

Patel, N. A., Goldsmith, P. F., Snell, R., Hezel, T. \& Xie, T. 1995, ApJ, 447, 721

Reid, M. J., Schneps, M. H., Moran, J. M., Gwinn, C. R., Genzel, R., Downes, D. \& Ronnang, B. 1988, ApJ, 330, 809

Saraceno, P., et al. 1996, A\&A, 315, L293

Schneps, M. H., Lane, A. P., Downes, D., Moran, J. M., Genzel, R. \& Reid, M. 1981, ApJ, 249,124

Snell, R. L., Loren, R. B. \& Plambeck, R. L. 1980, ApJ, 239, L17

Shu, F. H., Adams, F. C. \& Lizano, S. 1987, ARA\&A, 25, 23

Shu, F., Najita, J., Wilkin, F., Ruden, S. P. \& Lizano, S. 1994, ApJ, 429, 781

Slysh, V. I., Val'tts, I. E., Migenes, V., Fomalont, E., Hirabayashi, H., Inoue, M. \& Umemoto, T., 1999, ApJ, 526, 236.

Sugitani, K., Fukui, A., Mizuno, A. \& Ohashi, N. 1989, ApJ, 342, L87

Tofani, G., Felli, M., Taylor, G. \& Hunter, T. 1995, A\&AS, 112, 299

Wilking, B. A., Mundy, L., McMullin, J., Hezel, T. \& Keene, J. 1993, AJ, 106, 250

Wootten, A., et al. 1999, in Star Formation 1999, ed. T. Nakamoto, (Nagoya: Nagoya University), in press 
Table 1. Positions, velocities and proper motions of masers

\begin{tabular}{|c|c|c|c|c|c|c|c|}
\hline \multirow[b]{2}{*}{$\begin{array}{c}\mathrm{V}_{L S R} \\
\left(\mathrm{~km} \mathrm{~s}^{-1}\right)\end{array}$} & \multicolumn{2}{|c|}{ Position offset ${ }^{\mathrm{a}}$} & \multicolumn{4}{|c|}{ Proper motion ${ }^{\mathrm{b}}$} & \multirow[t]{2}{*}{ Maser } \\
\hline & $\begin{array}{c}\Delta \alpha \\
(\mathrm{mas})\end{array}$ & $\begin{array}{c}\Delta \delta \\
(\mathrm{mas})\end{array}$ & $\begin{array}{c}\dot{\alpha} \\
\left(\operatorname{mas~yr}^{-1}\right)\end{array}$ & $\begin{array}{c}\text { Error } \\
\left(\text { mas yr }^{-1}\right)\end{array}$ & $\begin{array}{c}\dot{\delta} \\
\left(\operatorname{mas~yr}^{-1}\right)\end{array}$ & $\begin{array}{c}\text { Error } \\
\left(\text { mas yr }^{-1}\right)\end{array}$ & \\
\hline-14.6 & 0.9 & 0.2 & -10.2 & 1.2 & -2.5 & 0.2 & A \\
\hline-10.0 & 529.4 & 191.6 & & & & & B \\
\hline-9.4 & 527.0 & 225.2 & 3.2 & 3.8 & 2.4 & 0.2 & C \\
\hline-9.1 & 529.0 & 190.1 & & & & & B \\
\hline-8.6 & 529.0 & 190.1 & & & & & B \\
\hline-8.0 & 528.4 & 190.0 & & & & & B \\
\hline-7.6 & 527.7 & 189.7 & & & & & B \\
\hline-7.6 & 529.3 & 190.0 & & & & & B \\
\hline-3.7 & 536.9 & 183.7 & & & & & B \\
\hline-1.9 & 535.0 & 182.3 & & & & & B \\
\hline-1.4 & 506.8 & 167.6 & -1.8 & 0.6 & -1.7 & 0.02 & B \\
\hline-1.0 & 509.1 & 168.6 & -0.3 & 0.5 & -2.2 & 0.1 & B \\
\hline-0.9 & 528.0 & 178.5 & & & & & B \\
\hline-0.5 & 515.3 & 168.1 & & & & & B \\
\hline-0.5 & 506.9 & 167.9 & & & & & $\mathrm{~L}$ \\
\hline-0.0 & 516.6 & 168.0 & & & & & $\mathrm{~L}$ \\
\hline 0.0 & 516.4 & 167.8 & & & & & $\mathrm{~L}$ \\
\hline 0.2 & 516.5 & 167.8 & & & & & $\mathrm{~L}$ \\
\hline 0.3 & 516.7 & 167.8 & & & & & $\mathrm{~L}$ \\
\hline 0.4 & 515.5 & 169.3 & 0.3 & 0.3 & -1.3 & 0.4 & $\mathrm{~L}$ \\
\hline 0.4 & 517.8 & 168.9 & & & & & $\mathrm{~L}$ \\
\hline 0.4 & 516.9 & 168.0 & & & & & B \\
\hline 0.4 & 507.0 & 168.0 & & & & & B \\
\hline 0.8 & 522.4 & 162.7 & & & & & $\mathrm{~L}$ \\
\hline 0.8 & 516.2 & 167.0 & & & & & $\mathrm{~L}$ \\
\hline
\end{tabular}


Table 1 - Continued

\begin{tabular}{|c|c|c|c|c|c|c|c|}
\hline \multirow[b]{2}{*}{$\begin{array}{c}\mathrm{V}_{L S R} \\
\left(\mathrm{~km} \mathrm{~s}^{-1}\right)\end{array}$} & \multicolumn{2}{|c|}{ Position offset $^{\mathrm{a}}$} & \multicolumn{4}{|c|}{ Proper motion $^{\mathrm{b}}$} & \multirow[t]{2}{*}{ Maser } \\
\hline & $\begin{array}{c}\Delta \alpha \\
(\mathrm{mas})\end{array}$ & $\begin{array}{c}\Delta \delta \\
(\mathrm{mas})\end{array}$ & $\begin{array}{c}\dot{\alpha} \\
\left(\operatorname{mas~yr}^{-1}\right)\end{array}$ & $\begin{array}{c}\text { Error } \\
\left(\operatorname{mas~yr}^{-1}\right)\end{array}$ & $\begin{array}{c}\dot{\delta} \\
\left(\operatorname{mas}^{-1} r^{-1}\right)\end{array}$ & $\begin{array}{c}\text { Error } \\
\left(\operatorname{mas~yr}^{-1}\right)\end{array}$ & \\
\hline 0.9 & 517.1 & 166.9 & & & & & $\mathrm{~L}$ \\
\hline 1.0 & 517.1 & 169.8 & 1.5 & 0.7 & -0.8 & 0.2 & $\mathrm{~L}$ \\
\hline 1.0 & 517.6 & 167.9 & & & & & $\mathrm{~L}$ \\
\hline 1.1 & 516.5 & 166.9 & & & & & $\mathrm{~L}$ \\
\hline 1.1 & 517.4 & 167.5 & & & & & $\mathrm{~L}$ \\
\hline 1.4 & 527.0 & 168.8 & 0.2 & 1.0 & 1.7 & 0.1 & B \\
\hline 1.5 & 526.3 & 161.2 & & & & & $\mathrm{~B}$ \\
\hline 2.0 & 528.5 & 175.8 & & & & & B \\
\hline 2.1 & 531.6 & 169.6 & 1.6 & 0.2 & 0.7 & 0.2 & B \\
\hline 2.5 & 523.0 & 168.7 & 1.0 & 0.4 & 0.8 & 0.4 & B \\
\hline 7.5 & 615.9 & 334.9 & 4.5 & 0.3 & 3.0 & 1.2 & $\mathrm{D}$ \\
\hline
\end{tabular}

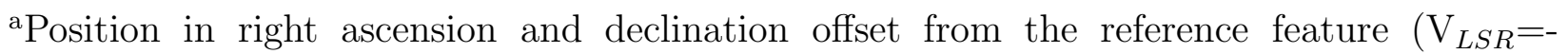
$14.6 \mathrm{~km} \mathrm{~s}^{-1}$ ), the estimated position of which is $\alpha_{J 2000}=21^{h} 40^{m} 41^{s} .791 \pm 0^{s} .004, \delta_{J 2000}=$ $58^{\circ} 16^{\prime} 11^{\prime \prime} 737 \pm 00^{\prime \prime} 030$

${ }^{\mathrm{b}} \mathrm{A}$ proper motion vector of $38.7 \mathrm{~km} \mathrm{~s}^{-1}$ at a position angle of $256^{\circ}$ has been added to the gross motions to achieve a zero mean (see text).

${ }^{\mathrm{c}} \mathrm{See}$ labels in Figure 2 


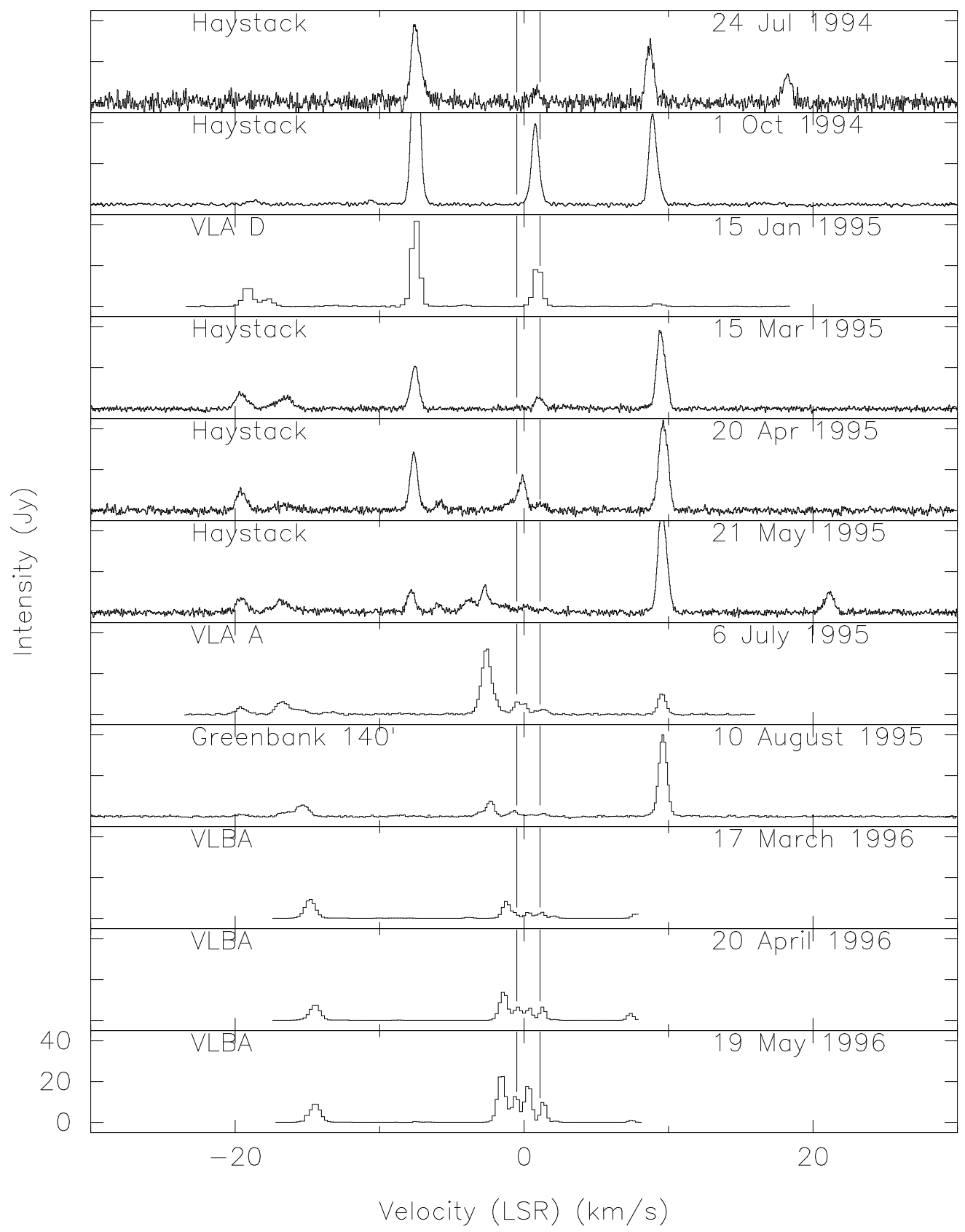

Fig. 1. - Total power spectra obtained at the Green Bank 140-foot and Haystack $37 \mathrm{~m}$ antennas and integrated VLA and VLBA spectra of water maser emission associated with IRAS 21391+5802. The maser features vary significantly in intensity on time scales greater than a month. Line-of-sight velocities may also change on time scales of months. The VLBA spectra covered the velocity range of -27 to $27 \mathrm{~km} \mathrm{~s}^{-1}$ but no emission was found at velocities less than $-14 \mathrm{~km} \mathrm{~s}^{-1}$ and greater than $8 \mathrm{~km} \mathrm{~s}^{-1}$. (The VLBA data are shown only for the range of spectral channels that display maser emission on the dates indicated). The vertical lines indicate the range of line-of-sight velocities of the masers occurring in the loop shown in Fig. 2. The systemic velocity of the source is $\sim 0 \mathrm{~km} \mathrm{~s}^{-1}$. 


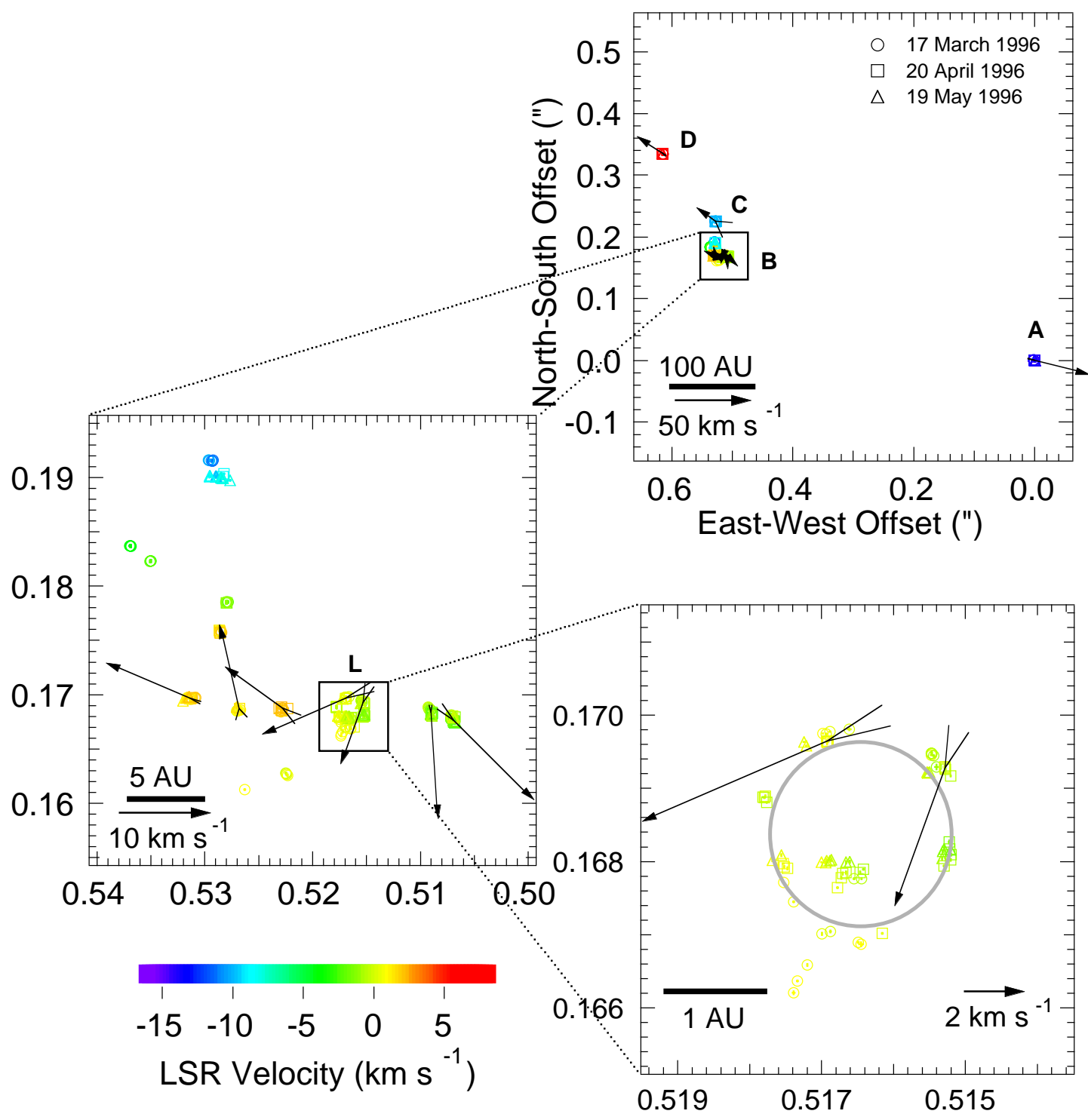

Fig. 2.- Maps of fitted water maser spots and maser feature proper motions from all 3 VLBA epochs superposed. Upper right: Positions and proper motions of the water masers are largely indicative of a bipolar outflow motion. The formal and systematic errors in these positions are much smaller than the size of the symbols in the plot. The $1 \sigma$ uncertainties in the direction of proper motion are indicated by the angle indicated at the tails of the arrows. The length of the cones imply $1 \sigma$ uncertainties in the magnitude of the proper motion. Line-of-sight velocities are indicated by symbol colors, according to the scale bar at the bottom left. The maser labeled A is the reference feature in all three epochs. Middle left: The proper motions and positions of the inner masers (В), are shown on an enlarged scale in the lower panel. Lower right: The masers roughly in the center of the B trace a nearly circular loop; the YSO is probably located at the center. The radius of the loop of masers is $\sim 1 \mathrm{AU}$. The loop most likely represents the inner radius of dust condensation in a shell of outflowing material around the YSO. 


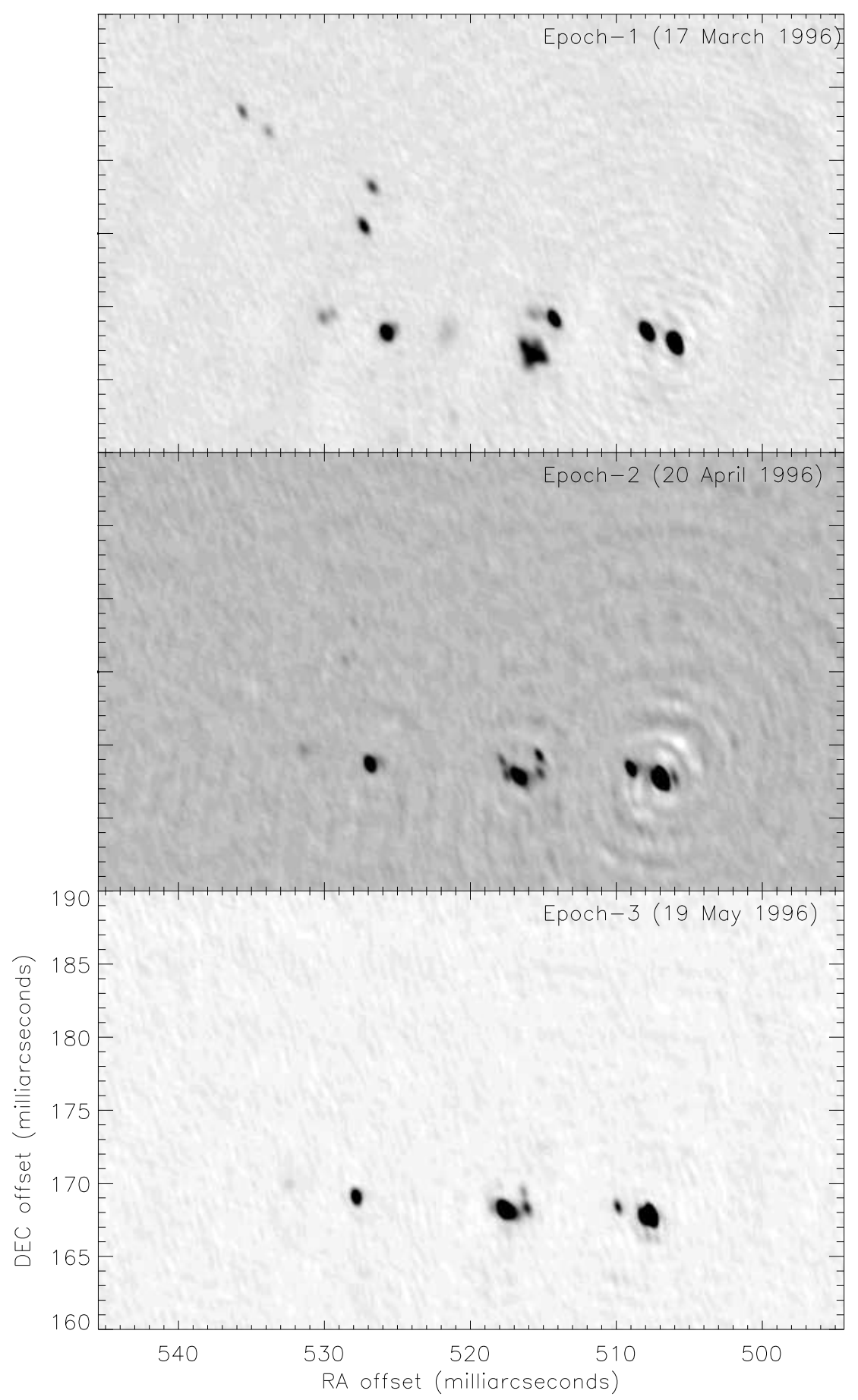

Fig. 3.- Integrated intensity images at three epochs, for emission between $\mathrm{V}_{L S R}=-4.6$ and $1.7 \mathrm{~km} \mathrm{~s}^{-1}$ within the central clump of masers. The overall variability of features in the spectra (Fig. 1) is consistent with changes among the maps. The masers near the position offset $\Delta \alpha=00^{\prime \prime} 516, \Delta \delta=0{ }^{\prime \prime} 168$ correspond to the loop pictured in Fig. 2. 


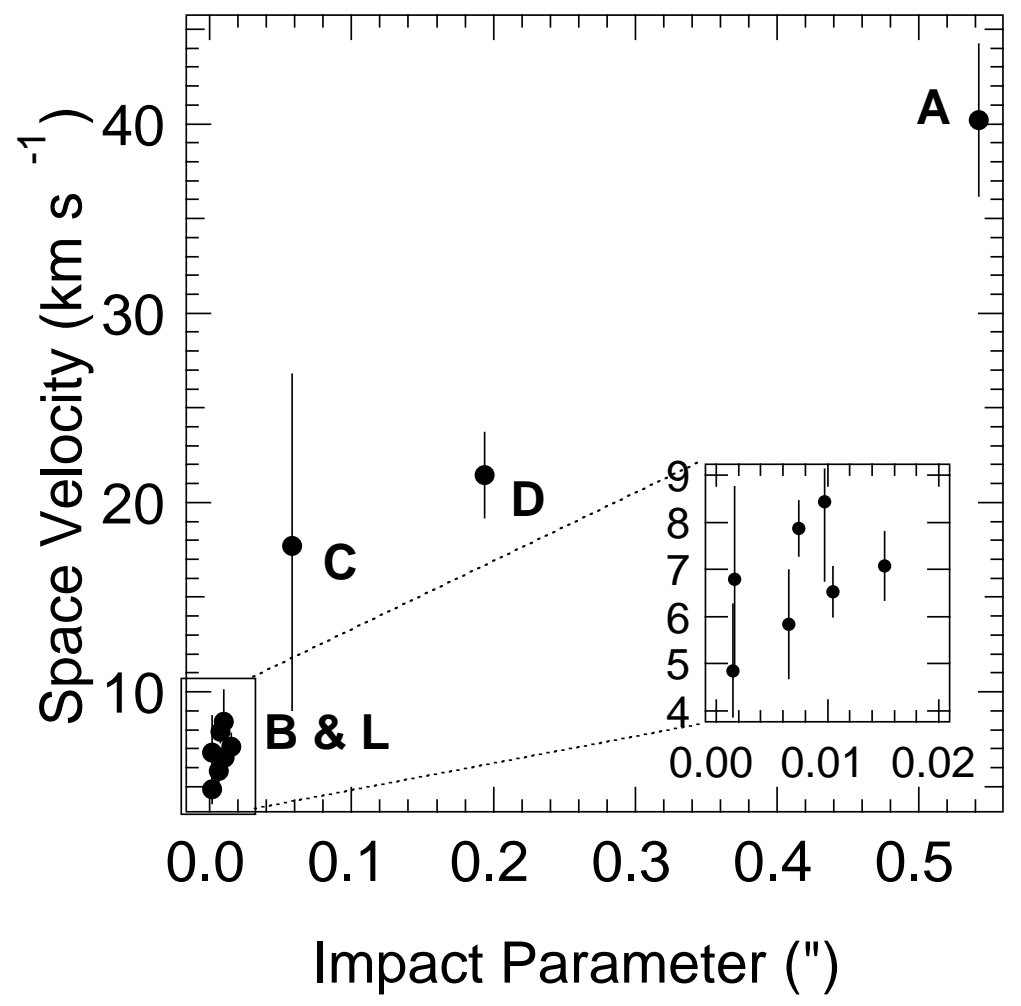

Fig. 4. - Variation of 3D space velocity as a function of distance from the position of the YSO (which is assumed to be at the center of the loop of masers shown in Fig. 2). This figure shows that 1) the masers that are more distant from the YSO, move relatively faster than the closer ones and 2) the dynamical time for the inner masers (B \& L) is less than that for the outer masers (A, C \& D). 